\title{
Discharge characteristics in inhomogeneous fields under air flow
}

\author{
Stephan Vogel, Joachim Holbøll \\ Technical University of Denmark
}

\begin{abstract}
This research investigates the impact of high velocity air flow on Partial Discharge (PD) patterns generated in strongly inhomogeneous fields. In the laboratory, a needle plane electrode configuration was exposed to a high electrical DC-field and a laminar air flow up to $22 \frac{\mathrm{m}}{\mathrm{s}}$. The needle was connected to a variable DC potential of up to $100 \mathrm{kV}$ over a grounded plate in order to trigger different corona modes. The impact of the air flow on the space charges created in the vicinity of the electrode is evaluated by means of PD measurements in time domain. The results indicate that the wind increases the frequency and magnitude of partial discharges in the vicinity of the electrode due to an increased rate of space charge removal around the tip of the needle and in the gap. The positive polarity shows higher dependency on air flow compared to the negative polarity. It is shown that positive breakdown streamer corona can be extinguished if wind speeds of more than $14.3 \frac{\mathrm{m}}{\mathrm{s}}$ are applied to the electrode.
\end{abstract}

\section{Introduction}

The frequency of upward lightning attachment to tall structures and wind turbines is biased by the height of the structure, topography of the surrounding environment, meteorological conditions and nearby lightning activity [1]. Recent publications indicate that also the impact of air flow may have a distinct impact on the distribution of space charges around the Lightning Protection System (LPS) of a structure which in turn may impact the frequency of the formation of upward lightning leaders [2]. Stationary objects, such as Franklin rods installed on towers, exposed to negative charged thunderclouds create positive charged space charges, which slowly propagate towards the cloud and hereby reduce the electric field around the extremities of the buildings. In the case of rotating wind turbines, generated space charges originating from the receptors are readily swept away from the electrode due to the dominant angular velocity of the rotation. Therefore, the resulting electric field around the leading edge of the wind turbine is expected to be higher as compared to stationary objects, leading to a theoretical higher rate of initiation of upward lightning leaders. This is of particular interest in winter lightning areas where frequent self-initiated upward lightning discharges are observed from tall structures.

The source of space charges are PD in air, also known as corona discharges, which are a well studied phenomenon and several publications describe studies on the general discharge behaviour of positive and negative corona [3] [4] [5]. Furthermore, the propagation of space charge in the atmosphere is well understood and studies of ion development in space and time can be found for example in [6] [7] [8]. Unlike electrons, ions are characterised by a relatively high mass and a comparably low propagation speed, which enables the interaction with air flow such as wind[9].

Only few publications, however, deal with the impact of air flow to corona discharges. F.D. Alessandro [10] studied the impact of wind to negative and positive corona currents at $3300 \mathrm{~m}$ above sea level on earthed pin needles created by the electric field of a thunderstorm where natural wind speeds up to $14 \frac{\mathrm{m}}{\mathrm{s}}$ were observed. In [11] the impact of air flow up to $16 \frac{\mathrm{m}}{\mathrm{s}}$ was investigated by means of a spark gap and a wind tunnel. The author showed that the onset corona voltage in his case was independent of the wind speed, however, there was an increase in the measured current while wind was applied to the gap. Similar findings are reported in [12]. The magnitude of corona current varied in these publications from approx. $1 u A$ to $300 u A$. All the above mentioned publications investigated DC corona currents.

To the knowledge of the authors, so far no systematic evaluation of PD pattern exposed to strong wind velocities up to $v=22 \frac{\mathrm{m}}{\mathrm{s}}$ has been performed.

The present work is structured as follows: At first the laboratory setup is described and some more details regarding the measurement setup is provided. Subsequently, negative and positive corona discharge patterns are illustrated and the impact of the air flow is discussed. The measurements were performed in the laboratory of the Technical University of Denmark.

\section{Experimental laboratory setup of DC- corona under air flow}

A high voltage DC source up to $100 \mathrm{kV}$ with $1 \mathrm{~mA}$ maximum current output is connected to a needle-plane spark gap with distance $d=20 \mathrm{~cm}$ and time domain PD measurements are performed with and without wind. The schematic of the setup is illustrated in Figure 1 and an overview of layout is presented in Figure 2. The purpose of this test is to investigate if air flow can change the patterns of discharges generated in the close vicinity of the needle tip. The needle had a diameter of $4 \mathrm{~mm}$ and an approximate tip diameter of 
$1 \mathrm{~mm}$. The PD measurements were performed by means of a Power Diagnostix ICM Measurement System with a bandwidth of $40 \mathrm{kHz}$ to $800 \mathrm{kHz}$, which detected the transient charge displacement across the spark gap via a measurement capacitor $C_{M}=400 p C$. Dead time between measurements was set to the lowest possible value of 2us. Lower noise threshold was set to $2 \%$ of the respective PD measurement scale. Furthermore, acoustic measurements for PD localisation were performed by means of an ultrasonic PD detector. A bleeding resistor $R_{B}=330 G \Omega$ was added to ensure save discharge of the measurement setup when powered down. The DC voltage of $100 k V$ applied to a needleplane electrode configuration of $20 \mathrm{~cm}$ represents near breakdown conditions, so all corona modes could be studied. The wind source was a modified industrial fan for buildings which produced a laminar air flow on an rectangular air-outlet surface of $61 \mathrm{~cm}$ times $30 \mathrm{~cm}$. The needle electrode was mounted $40 \mathrm{~cm}$ from the outlet of the fan and a maximum wind speed of $22 \frac{\mathrm{m}}{\mathrm{s}}$ was measured in the plane of the electrode.

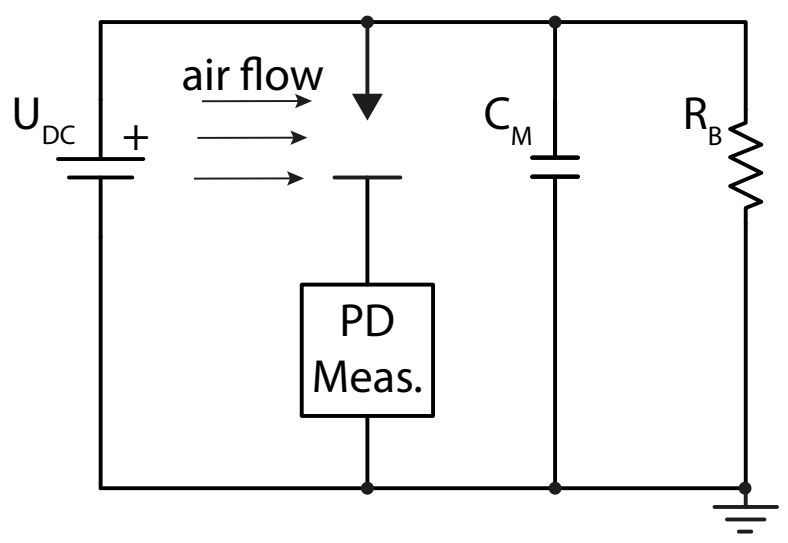

Fig. 1 - Circuit diagram for PD measurement on needle-plane spark gap exposed to wind flow. The DC voltage $U_{D C}$ is varied between 0 and $90 \mathrm{kV}$. The air flow was $22 \frac{\mathrm{m}}{\mathrm{s}}$ at $n=1500 \mathrm{rpm}$. Partial discharges were measured with the low impedance PD measurement impedance in series with electrode gap and across the measurement capacitor $C_{M}$. The bleeding resistor acts as safety equipment.

\subsection{Methodology of measurements}

The methodology for the PD measurements is the following. Constant DC voltage was applied to the needle tip and the PD patterns were measured during 100 seconds. From $t=0 s$ to $t=20 \mathrm{~s}$ there was no air movement in the gap. At $t=20 \mathrm{~s}$ the industrial fan was turned on and the wind speed increased in the electrode configuration. The fan accelerated linearly from $n=0 \mathrm{rpm}$ to $n=1500 \mathrm{rpm}$ in ten seconds. From $t=30 \mathrm{~s}-40 \mathrm{~s}$ the maximum wind speed of $v=22 \frac{\mathrm{m}}{\mathrm{s}}$ was applied. At $t=40 \mathrm{~s}$ the fan was turned off and the fan speed linearly decreased within ten seconds. It needs to be noticed that air movement continued slightly

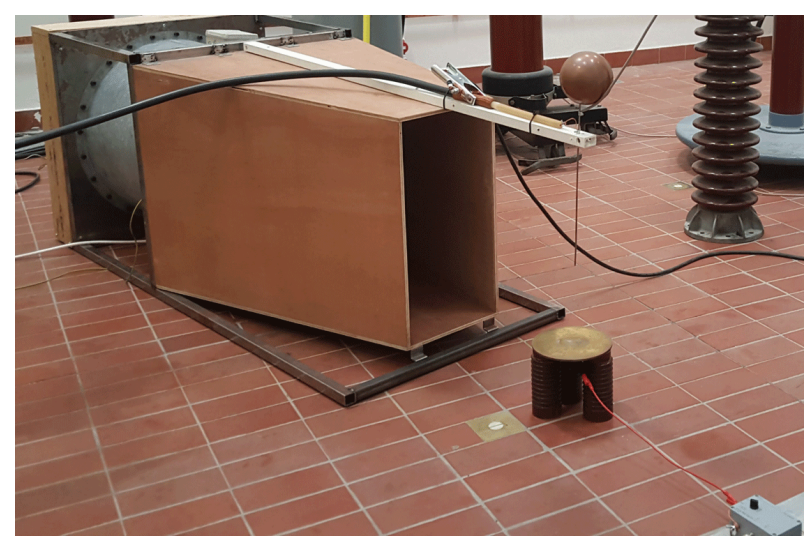

Fig. 2 - Fan and needle-plane electrode configuration with PD measurement equipment.

Table 1 - Rotational speed vs. wind speed of industrial fan

\begin{tabular}{ll}
\hline $\begin{array}{l}\text { Rotational Speed } \\
{[\text { rpm }]}\end{array}$ & $\begin{array}{l}\text { Wind Speed } \\
{\left[\frac{m}{s}\right]}\end{array}$ \\
\hline 250 & 3.4 \\
500 & 7.1 \\
750 & 10.5 \\
1000 & 14.2 \\
1250 & 17.5 \\
1500 & 21.8 \\
\hline
\end{tabular}

in the gap after the fan reached idle state due to the inertia of the air. At $t=60 \mathrm{~s}$, the fan was switched-on again and the processed was repeated in similar fashion. Both positive and negative polarities at the needle were evaluated and the results are presented the Figure 3 and 4. Each illustration contains nine diagrams which show the behaviour at discrete voltage levels. On the top of each illustration, the time dependent rotational speed of the fan is illustrated. The correlation between steady state wind speed and rotational speed is given in Table 1.

\subsection{Negative corona}

Figure 3 illustrates the impact of air flow to the PD patterns under negative polarity at the needle. The corona onset was determined for the negative polarity at $U_{D C}=3 \mathrm{kV}$. Compared to the positive polarity, the PD started earlier since initiating electrons are readily available from the negatively charged electrode. When increasing the applied voltage, the negative corona experiences three different modes, which are Trichel streamer (or Trichel pulses), pulseless negative glow, and negative streamer. Each mode has characteristic properties as described by [13]. For further information regarding corona modes please refer to the given source.

Trichel streamer corona was identified between $U_{D C}=3 k V-40 k V$, pulseless negative glow between $U_{D C}=50 \mathrm{kV}-60 \mathrm{kV}$ and negative streamer from $U_{D C}=70 \mathrm{kV}$. The main discharge band of negative PD are located between a few picocoulombs 

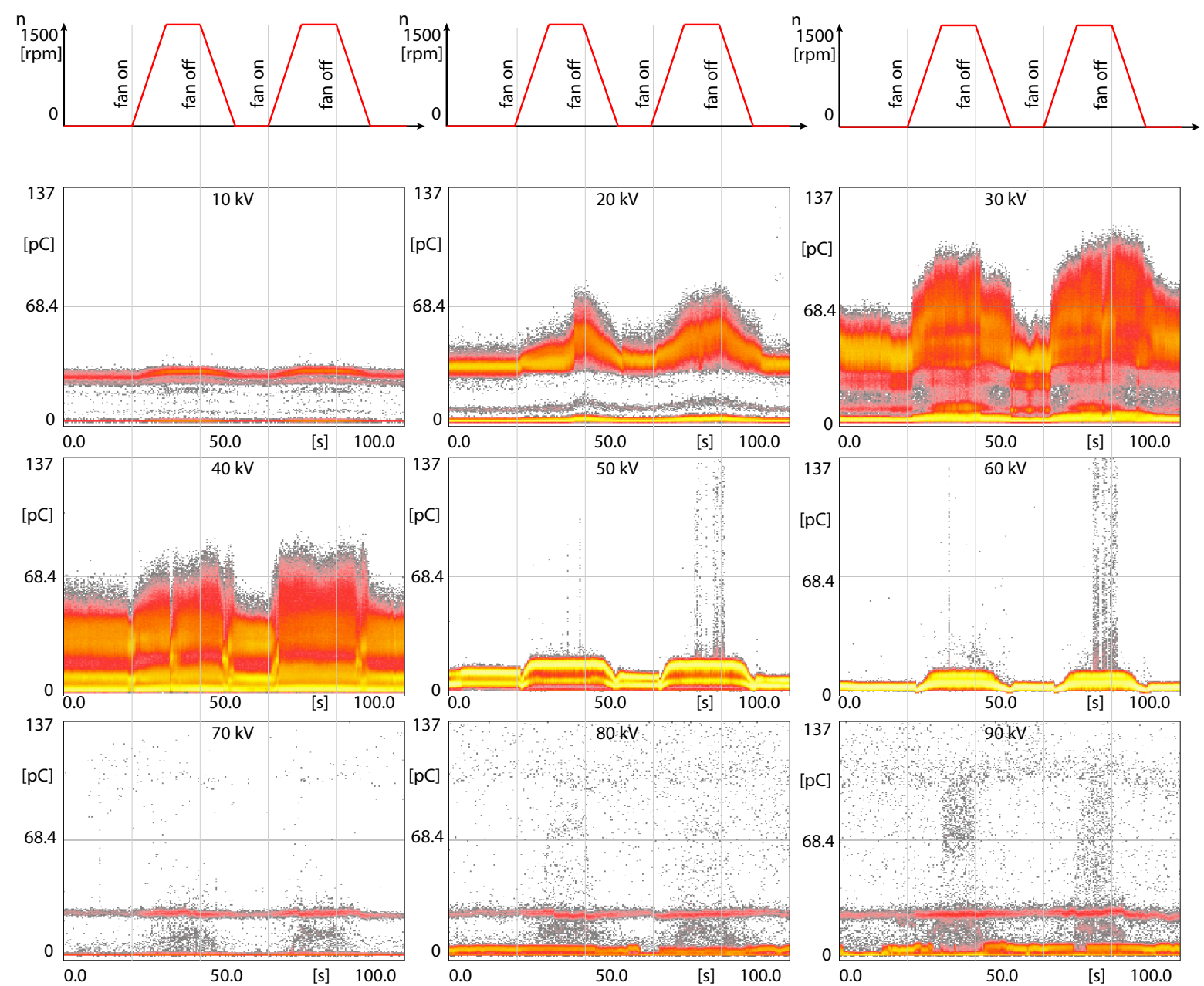

Fig. 3 - Negative polarity: 100 seconds of PD measurements with air flow from $t=20 \mathrm{~s}-50 \mathrm{~s}$ and $t=60 \mathrm{~s}-90 \mathrm{~s}$. Trichel streamer corona mode from $U_{D C}=3 k V-40 k V$, pulseless negative glow corona from $U_{D C}=50 k V-60 k V$, streamer corona from $U_{D C}=70 k V-90 k V$.

and 137 picoculomb. At the higher DC voltages above $U_{D C}=70 \mathrm{kV}$, there were also repetitive discharges observed which were characterised by a low frequency and a charge magnitude of up to several nanocoulombs (Frequency here is understood as pulse repetition rate). These results are related to negative streamer modes which reached further into the gap.

As can be seen in Figure 3, at the negative polarity, the air flow increases mainly the magnitude of the discharges. In the Trichel streamer mode between $U_{D C}=3 \mathrm{kV}-$ $40 \mathrm{kV}$, the maximum amplitude increases between $5 \%$ and $100 \%$ depending on the DC voltage. Since the discharges are spread within a wider band, also the frequency of discharges increases. When air flow is applied in the negative glow corona state between $U_{D C}=$ $50 k \mathrm{~V}-60 \mathrm{kV}$, the characteristic high frequency PD pattern increase slightly in magnitude and various larger discharges are superimposed on the measurement. The air flow changes the PD pattern from pulseless negative glow to negative streamer corona. From $U_{D C}=70 \mathrm{kV}-$
$90 \mathrm{kV}$, the air flow increases slightly the frequency and magnitude of the PD pattern.

\subsection{Positive corona}

Four different positive corona modes can be distinguished which are burst corona, onset streamer, glow corona, and breakdown streamer [13]. The measurement results are illustrated in Figure 4. Corona onset for the positive polarity was determined to $U_{\text {onset }}=12 \mathrm{kV}$. Burst corona and onset streamer corona can appear within a very short voltage range [13] and were determined simultaneously between a voltage range of $U_{D C}=12 k \mathrm{~V}-14 \mathrm{kV}$. Compared to the negative polarity, the PD of the initial positive corona mode was characterised by a higher charge magnitude (up to $Q=500 p C$ ) and a lower frequency. This is in good agreement with the gas discharge theory since continious availability of starting electrons at the negative polarity enable more frequent discharges with a lower amplitude, whereas PD at positive biased electrodes trigger at random electrons in the ambient air [14]. The glow corona mode started 

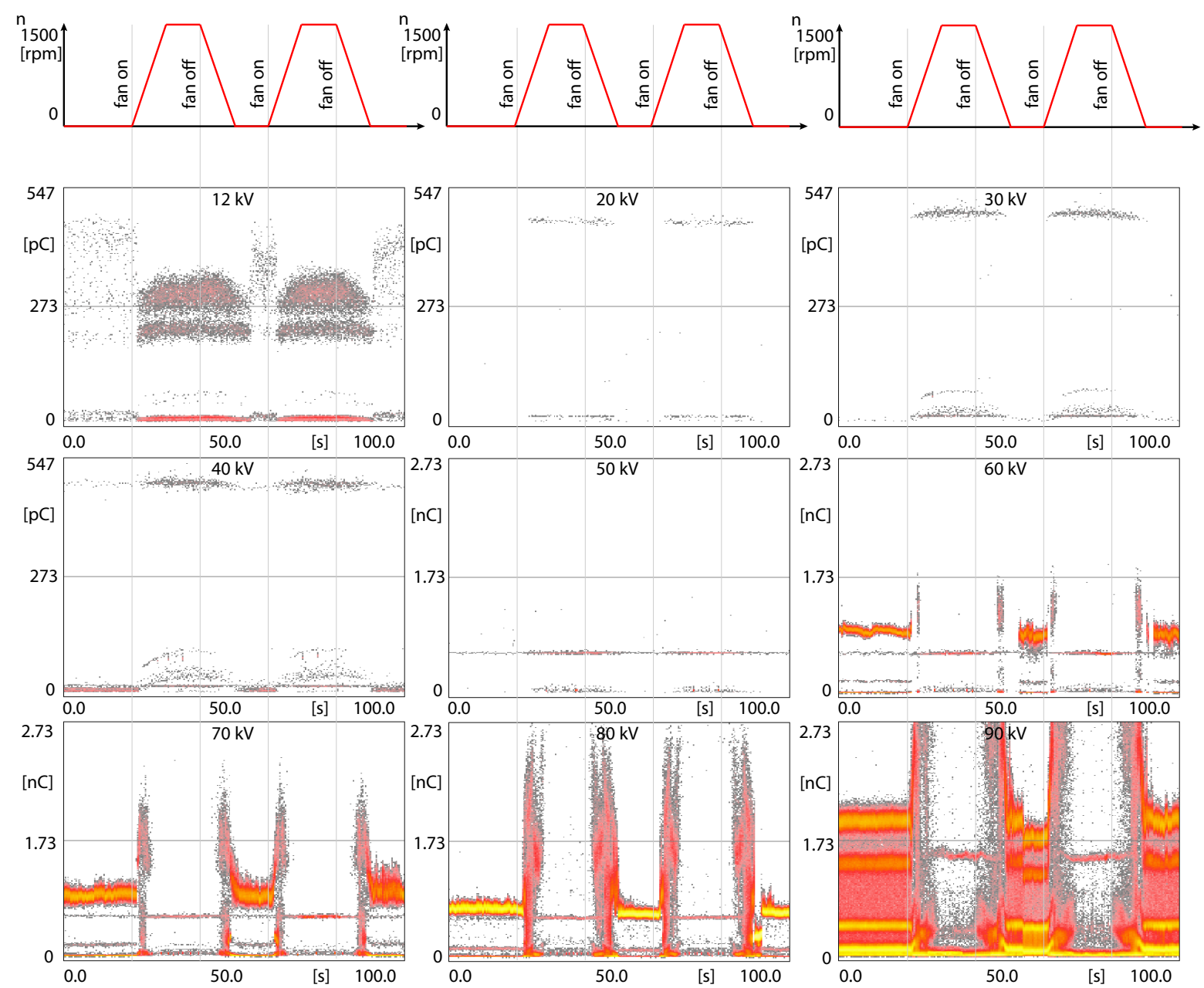

Fig. 4 - Positive polarity: 100 seconds of PD measurements with air flow from $t=20 \mathrm{~s}-50 \mathrm{~s}$ and $t=60 \mathrm{~s}-90 \mathrm{~s}$. Burst corona and onset streamer corona mode from $U_{D C}=12 k V-14 k V$, positive glow corona from $U_{D C}=14 k V-50 k V$, breakdown streamer corona from $U_{D C}=60 \mathrm{kV}-90 \mathrm{kV}$.

at approximately $U_{D C}=14 k V$ and continued until $U_{D C}=50 \mathrm{kV}$. Few positive glow corona discharges were detected with the PD-measurement equipment. The reason for this appearance is most likely the high frequency of glow corona pulses in DC fields of several Megaherz [5]. With an upper cut-off frequency of $800 \mathrm{kHz}$ from the PD measurement equipment, many pulses may be not able to be detected. Breakdown streamer corona starts at $U_{D C}=60 \mathrm{kV}$ and continues until $U_{D C}=90 \mathrm{kV}$. Notice, the y-axis scale in Figure 4 changes from $Q=547 p C$ to $Q=2.73 n C$. With increasing voltage, the frequency of the pulses as well as the magnitude increases.

The impact of the air flow is more pronounced at positive polarity compared to negative polarity. The strongest impact can be seen at burst corona/onset streamer corona and breakdown streamer corona as can be seen in Figure 4. The reason for the observation is that streamers propagate further into the gap and are not only contained around the vicinity of the gap. When partial discharge channel propagate further into the gap, also the air flow has higher impact since it can influence the movement of charged ions.

In the case of $U_{D C}=12 \mathrm{kV}$ illustrated in Figure 4, the frequency of pulses increase significantly, whereas the magnitude of pulses decreases slightly. Glow corona from $U_{D C}=20 \mathrm{kV}-U_{D C}=30 \mathrm{kV}$ could not be measured with the PD-measurement equipment without air flow, however, when air flow was applied, pulses clearly could be detected. A possible explanation is that the air flow removes the shielding ions from the electrode, leading to discharges which are higher in magnitude and lower in frequency. From $U_{D C}=60 \mathrm{kV}-U_{D C}=90 \mathrm{kV}$ breakdown streamer corona appears. It can be observed that the magnitude of PD increase while the fan is starting up and the air flow is increasing in the gap. If the air flow reaches a critical wind speed, the occurrence of PD stops. The breakdown streamer corona is extinguished and possibly transformed into a reduced glow corona discharge pattern which takes place only in the vicinity of the electrode. 


\section{Positive breakdown streamer corona extinguished by} high air flow
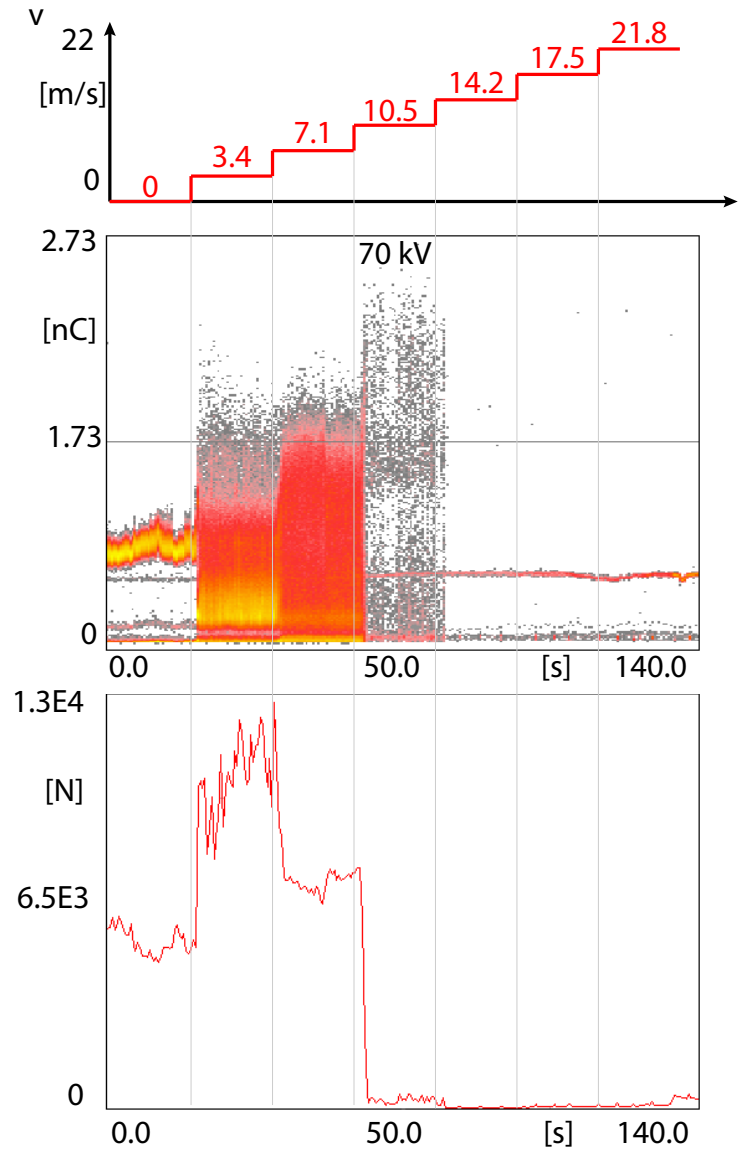

Fig. 5 - Breakdown streamer corona exposed to variable amount of air flow. The magnitude of PD increases with increasing air flow and the frequency (number of discharges) of PD increases first at $v=3.4 \frac{\mathrm{m}}{\mathrm{s}}$ and decreases from $v=3.4 \frac{\mathrm{m}}{\mathrm{s}}$ until breakdown streamer corona is extinguished.

In order to see the impact of air flow to the breakdown streamer corona, a separate experiment was performed where the wind magnitude was varied at a steady DC voltage of $U_{D C}=70 \mathrm{kV}$. As can be seen in Figure 5, already at slight wind speeds of $v=3.4 \frac{\mathrm{m}}{\mathrm{s}}$ the magnitude of PD discharges increases as well as the frequency of the discharges. At $v=7.1 \frac{\mathrm{m}}{\mathrm{s}}$, the magnitude does not vary distinctly; however, the frequency (Number of discharges $N)$ of the PD decreases. At $v=10.5 \frac{\mathrm{m}}{\mathrm{s}}$, the magnitude of PD is further increased but the amount of discharges are significantly reduced. From $v=14.2 \frac{\mathrm{m}}{\mathrm{s}}$, the breakdown streamer corona is extinguished. There remains a high frequency PD pattern which is of similar nature of the glow corona mode.

\subsection{Comparison between the impact of air flow from positive and negative polarity}

Following conclusions can be drawn from the laboratory experiment:

1. Corona caused by negative polarity is characterised by lower charge magnitudes compared to positive polarity, however:

2. The frequency of the discharges at negative polarity is much higher.

3. The corona discharge pattern of the positive polarity is more influenced by the effect of wind compared to negative polarity, especially for DC voltages over $U_{D C}=60 \mathrm{kV}$.

4. Positive breakdown streamer corona can be extinguished or reduced to glow corona mode when higher wind speeds than $v=14.2 \frac{\mathrm{m}}{\mathrm{s}}$ are exposed to the electrode.

\section{Conclusion}

In this paper, the impact of air flow on discharge characteristics in inhomogeneous fields was investigated and clearly confirmed. PD measurements were performed and the impact of wind on the different corona modes was demonstrated. The time domain PD pattern of the positive needle polarity shows higher dependence on air flow compared to the negative polarity. Air flow increases the magnitude and frequency of corona discharges in the needle-plane electrode configuration, proofing the importance of the space charges in the gap and their displacement by means of the air flow. An exception is the positive breakdown streamer corona $U_{D C}>60 k \mathrm{~V}$ which is extinguished by air flow from $v=14 \frac{\mathrm{m}}{\mathrm{s}}$.

\section{Acknowledgements}

The research has been partly funded by the Danish Energy Agency through the Energy Technology Development and Demonstration Program (EUDP), as part of the ELITE [15] project.

[1] Stephan Vogel, Joachim Holbøll, Javier López, Anna Candela' Garolera, and Søren Find Madsen. Lightning Attachment Estimation to Wind Turbines by Utilizing Lightning Location Systems. In International Lightning Detection Conference, 2016.

[2] Joan Montonaya, Oscar van der Velde, and Earl Williams. Lightning discharges produced by wind turbines. Journal of Geophysical Research, 119:1455-1462, 2014.

[3] Leonard B. Loeb and Arthur F. Kip. Electrical discharges in air at atmospheric pressure: The nature of the positive and negative point-to-plane coronas and the mechanism of spark propagation. Journal of Applied Physics, 10(3):142-160, 1939.

[4] R. Morrow. Theory of negative corona in oxygen. Physical Review A, 32(3):1799-1809, 1985.

[5] R Morrow. The theory of positive glow corona. Journal of Physics D: Applied Physics, 30(22):3099-3114, 1999. 
[6] Serge Soula and Serge Chauzy. Multilevel measurement of the electric field underneath a thundercloud: 2. Dynamical evolution of a ground space charge layer. Journal of Geophysical Research, 96(D12):22327, 1991.

[7] Marley Becerra, V. Cooray, S. Soula, and S. Chauzy. Effect of the space charge layer created by corona at ground level on the inception of upward lightning leaders from tall towers. Journal of Geophysical Research Atmospheres, 112(12):1-11, 2007.

[8] Marley Becerra. Glow corona discharges and their effect on lightning attachment: Revisited. 2012 31st International Conference on Lightning Protection, ICLP 2012, pages 2-6, 2012.

[9] Stephan Vogel, Javier Lopez, Joachim Holbøll, Søren Find Madsen, Anna Candela Garolera, and Kim Bertelsen. Numerical Simulation of the effect of wind removing the corona space charge over grounded structures under thunderstorm conditions. In International Conference on Lightning \& Static Electricity, 2015.

[10] F. D'Alessandro. Experimental study of the effect of wind on positive and negative corona from a sharp point in a thunderstorm. Journal of Electrostatics, 67(2-3):482-487, 2009.

[11] David F Ariza, Oscar R Montero, Francisco J Román, and Oscar F Escobar. Influence of the $\mathrm{W}$ ind for $\mathrm{P}$ ositive $\mathrm{C}$ orona in a $\mathrm{S}$ ingle- needleelectrode on $\mathrm{T}$ hunderstorm-like $\mathrm{C}$ onditions. In International Symposium on Lightning Protection (SIPDA), pages 101-106, 2011.

[12] F. Avella, J. Díaz, D. Ariza, O. Escobar, and F. Román. Avella, F., Díaz, J., Ariza, D., Escobar, O., \& Román, F. (2012). Influence of thunderstorm-like wind velocities on point discharge corona currents. 2012 31st International Conference on Lightning Protection, ICLP 2012, 0-3. https://doi.org/10.1109/ICLP.2. In 31st International Conference on Lightning Protection, ICLP, pages 0-3, 2012.

[13] N. Giao Trinh. Partial Discharge XIX: Discharge in Air Part I: Physical Mechanisms. IEEE Electrical Insulation Magazine, 11(2):23-29, 1995.

[14] Loeb. Electrical coronas. University of California Press, Cambridge University Press, 1965.

[15] S F Madsen, S Vogel, J Lopez, A C Garolera, and $\mathrm{K}$ Bertelsen. ELITE - ENHANCED LIGHTNING EFFECTS TESTING CAPABILITIES. In International Conference on Lightning and Static Electricity (ICOLSE), pages 1-8, 2015. 